\title{
EMERGENCY TREATMENT IN GYNECOLOGY USING DIFFERENT METHODS OF SURGERY
}

\author{
Iuliia Pikul $\bowtie$ \\ Department of Obstetrics, Gynecology and Perinatology ${ }^{l}$ \\ pikul_julia@ukr.net \\ Oleksandra Lubkovska \\ Department of Obstetrics, Gynecology and Perinatology ${ }^{l}$ \\ ${ }^{1}$ Ukrainian State Institute of Reproductology \\ Shupyk National Healthcare University of Ukraine \\ 9 Dorohozhytska str., Kyiv, Ukraine, 04112
}

$\triangle$ Corresponding author

\begin{abstract}
The aim. Improving surgical treatment of urgent genital pathology based on comparing the effectiveness of different methods of surgery.

Materials and methods. 170 women were selected and divided into three groups. Group 1 included women with acute pelvic inflammatory disease (aPID); to 2nd group - 60 women with intra-abdominal bleeding (IAB); to 3rd group - 50 women with impaired blood supply to tumors of the internal genitalia (uterus and ovaries) (IBSTIG). Each group was divided into two equivalent subgroups depending on the method of treatment: laparoscopic or laparotomy.

During the study, all examined patients had a medical history, gynecological examination, ultrasound examination, surgical treatment by laparoscopy or laparotomy.

Results. The average duration of laparoscopic operations in patients in subgroup 1.1 was $50.4 \pm 7.2$ minutes and $80.3 \pm 10.1 \mathrm{mi}-$ nutes in subgroup 1.2 - with laparotomy method. Intraoperative blood loss was significantly lower in subgroup 1.1 compared with subgroup $1.2(210.7 \pm 31.4 \mathrm{ml}$ and $375.9 \pm 44.5 \mathrm{ml})(p<0.01)$. In the postoperative period in subgroup 1.1, which included women with emergencies due to aPID and in which operations were performed laparoscopically, the maximum temperature rise after surgery was $37.6 \pm 0.04{ }^{\circ} \mathrm{C}$, which was significantly lower than in subgroup 1.2 , which included women with emergencies due to aPID and in which operations were performed by laparotomy, this figure was $38.0 \pm 0.08{ }^{\circ} \mathrm{C}(p<0.001)$. The mean duration of the postoperative period in patients of subgroup 1.1 was significantly shorter compared to subgroup $1.2(7.2 \pm 0.6$ and $10.2 \pm 1.0$ days $)(p<0.05)$.

The average duration of operations in subgroup 2.1 was significantly shorter than in subgroup $2.1(39.8 \pm 2.1$ and $59.1 \pm 2.5 \mathrm{mi}-$ nutes, respectively) $(p<0.001)$. Intraoperative blood loss was on average significantly lower in subgroup 2.1 (189.1 \pm 19.8 and $388.5 \pm 37.2 \mathrm{ml}$, respectively) $(p<0.05)$.

In subgroup 3.1, which included women with IBSTIG and underwent surgical treatment by laparoscopy, the average duration of operations was significantly shorter than in subgroup 3.2, where such women with such pathology received surgical treatment by laparotomy (62.1 \pm 4.9 and $89.6 \pm 7.8$ minutes, respectively) $(p<0.01)$. Blood loss was significantly lower in subgroup 3.1 in contrast to subgroup $3.2(101.7 \pm 10.1 \mathrm{ml}$ and $283.3 \pm 12.9 \mathrm{ml}$, respectively) $(p<0.001)$.

Conclusions. The use of laparoscopy for the treatment of urgent genital pathology could increase the effectiveness of surgical treatment. In addition, this method reduces the duration of surgery and surgical blood loss. As a result, it makes it possible to improve the postoperative period.
\end{abstract}

Keywords: laparoscopy, intra-abdominal bleeding, pelvic inflammatory disease, postoperative period.

DOI: $10.21303 / 2504-5679.2022 .002276$

\section{Introduction}

Maintaining a woman's reproductive health is a major challenge in gynecology. One of the reasons for its violations is untimely diagnosis and incorrect tactics of treatment of urgent conditions that require immediate surgical treatment. Such conditions are characterized by an ambiguous clinical picture, difficult early diagnosis, lack of positive dynamics for conservative treatment, rapid deterioration, frequent surgical treatment, complications in the postoperative period. Often, timely surgical treatment is the key to restoring both reproductive and physical health of women $[1,2]$. 
There are various methods of surgical treatment, but clinicians are paying more and more attention to endoscopic technologies. These include laparoscopy and consider it the most minimally invasive method of surgical treatment. Therefore, obstetricians and gynecologists often try to use in gynecology for the treatment of such urgent conditions as acute inflammatory diseases of the pelvic organs, intra-abdominal bleeding, tumors with acute disruption of their blood supply. Such conditions include ectopic pregnancy, apoplexy and trophic disorders of the ovaries. Clinical manifestations of urgent conditions in gynecology depend on the pathology of the reproductive system and the amount of blood loss [3, 4].

In addition, endoscopic methods could clarify the presence of the main gynecological pathology and choose the optimal amount of surgical treatment.

However, despite the significant number of publications on the use of laparoscopy in gynecology, it remains a debatable question about its effectiveness compared to surgical treatment by laparotomy.

The aim is to improve the tactics of treatment of urgent genital pathology based on the comparison of the effectiveness of different methods of surgical intervention.

\section{Materials and methods}

The study was conducted from 2018 to 2020 on the basis of the gynecological department of the Kyiv City Clinical Hospital No. 6. To achieve this goal, 170 women were selected and divided into three groups. Group 1 included 60 women with acute aPID; up to 2 groups - 60 women with IAB; up to 3 groups - 50 women with IBSTIG. The age of the studied women of the I group ranged from 25 to 37 years. The average age was $29.6 \pm 2.0$ years. The age of the studied women of the II group ranged from 24 to 35 years. The average age is $28.9 \pm 2.5$ years. The age of the studied women of group III ranged from 35 to 47 years. The mean age of patients in group III was $41.4 \pm 2.7$ years. In turn, each group was divided into two equivalent subgroups depending on the method of treatment. Thus, the treatment of women in subgroups 1.1, 2.1, 3.1 consisted in the use of modern endoscopic technologies, namely surgical treatment by laparoscopy. In subgroups 1.2, 2.2, 3.2, in contrast to the previously mentioned subgroups, surgical treatment of emergencies by laparotomy was performed.

The study was conducted in accordance with the Declaration of Helsinki according to the conclusion of the Commission on Ethics of the National University of Health of Ukraine named after P. L. Shupyk (minutes No. 2 of 09.02.2017). Informed consent to participate in the study was obtained from all women.

The analysis of the groups showed that the average age of women was $29.6 \pm 0.5$ years $(p<0.05)$. According to the gynecological anamnesis, the results of the identified gynecological and somatic diseases of the selected women in the subgroup comparison did not differ significantly.

On admission to the hospital, $82 \%$ of patients (i.e., 140 women) complained of significant lower abdominal pain, most with irradiation to the rectum and lumbar region. $56 \%$ of women (95 patients) had complaints of weakness and malaise, and $38 \%$ of patients ( 65 women) had fever. In addition, $40 \%$ (68) of the surveyed women had dyspeptic symptoms, and $10 \%$ (17 women) - dysuric.

During the study, all examined patients had a medical history, gynecological examination, ultrasound examination, surgical treatment by laparoscopy or laparotomy, as well as morphological examination of the material obtained during surgery.

Laparoscopy was performed using Olympus optical systems and instruments, Japan.

Ultrasound was performed using the device company «Aloka SSD 3500» (Japan), transabdominal and transvaginal access. During the ultrasound, the features of the pelvis were evaluated, in particular, the uterus, fallopian tubes, ovaries, and ectopic uterus.

Statistical processing of the study results was performed using Statistica and Microsoft Office Excel. Statistical significance was assessed using Student's t-test and $\chi^{2}$. Differences were considered statistically significant at $p<0.05$.

\section{Results}

Analyzing two subgroups of group 1, which included women with emergencies due to aPID, it was found that the most common complaints were fever, namely in $100 \%$ (60 patients) and 
lower abdominal pain - $98.3 \%$ (i.e., in 59 patients). Complaints such as: weakness was observed in $60 \%$ of patients (in 36 patients), bowel incontinence - in $40 \%$ of women (in 24 patients), dysuric phenomena $-6.7 \%$ of patients (in 4 patients).

In addition, it was found that among patients 1.1 subgroup admitted to the hospital at the prehospital stage of treatment did not receive $73.3 \%$ (22) of women, and among 1.2 subgroup $76.7 \%$ (i.e., 23) $(p>0.05)$. At the same time, in subgroup $1.126 .7 \%$ (i.e., 8 ) and in subgroup 1.2 $23.3 \%$ (7) patients underwent self-treatment at home $(p>0.05)$.

The study found that most patients in group 1 had unilateral or bilateral pyosalpinx (Fig. 1), and $53.3 \%$ (32) of women had a complication of the underlying diagnosis, namely pelvioperitonitis.

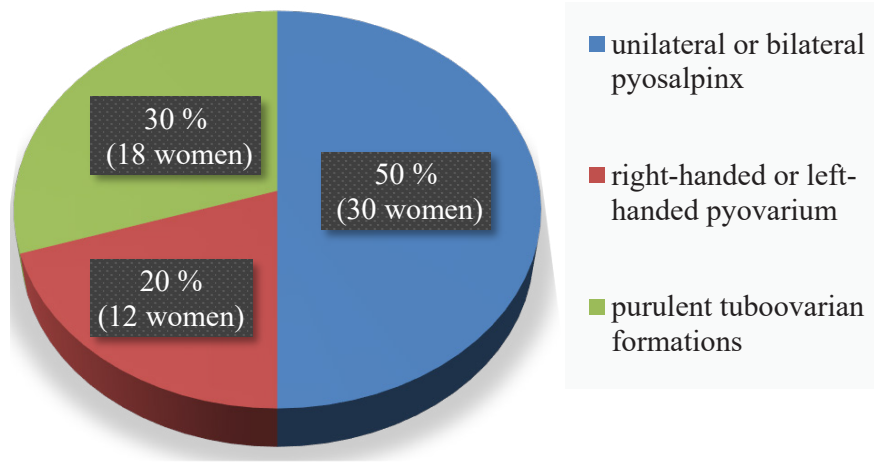

Fig. 1. The structure of pathological conditions in women with aPID

All patients underwent surgery after previous detoxification and symptomatic therapy. The duration of preoperative preparation was, on average, $0.7 \pm 0.1$ days in 1.1 and $0.5 \pm 0.1$ days in subgroup $1.2(p>0.05)$ and was determined individually depending on the stage of acute inflammatory process, the severity of intoxication.

In order to determine the least invasive and most effective method of treatment of urgent conditions in gynecology in subgroup 1.130 patients were operated laparoscopically, and in 1.2 subgroup 30 women - laparotomy. When analyzing the volume of surgical interventions, it should be noted that tubectomy was performed in 17 women (56.7 \%) in 1.1 subgroup and 13 women (43.3 \%) in 1.2 subgroup, which did not differ significantly $(p>0.05)$, ovariectomy in 5 patients $(16.7 \%)$ of 1.1 subgroup and in 7 patients $(23.3 \%)$ of 1.2 subgroup $(p>0.05)$; removal of tuboovarian formation was performed in 8 patients $(26.7 \%)$ of 1.1 subgroup and in 10 women $(33.3 \%)$ of 1.2 subgroup $(p>0.05)$.

After analyzing the average duration of surgery and intraoperative blood loss, it was found that they were significantly better in subgroup 1.1, where the laparoscopic method is used (Table 1).

Table 1

Characteristics of different methods of surgery in women with aPID

\begin{tabular}{cccc}
\hline Indicators & Subgroup 1.1 $(\boldsymbol{n}=\mathbf{3 0})$ & Subgroup 1.2 $(\boldsymbol{n}=\mathbf{3 0})$ & $\boldsymbol{P}$ \\
\hline Duration of operation, min & $50.4 \pm 7.2$ & $80.3 \pm 10.1$ & $p<0.05$ \\
Intraoperative blood loss, $\mathrm{ml}$ & $210.7 \pm 31.4$ & $375 \pm 44.5$ & $p<0.01$
\end{tabular}

After analyzing the postoperative period, it was found that the parameters by which it was evaluated were significantly better in subgroup 1.1, which used a laparoscopic method of treatment of acute aPID (Table 2).

Therefore, the use of laparoscopy in surgical treatment of aPID could improve the results of both surgical treatment and the postoperative period.

Subgroups 2.1 and 2.2 included 30 women with intra-abdominal bleeding as a result of ectopic pregnancy (63.3\% (19) in subgroup 2.1 and $73.3 \%(22)$ in 2.2) and apoplexy of the right or left ovary $(36.7 \%(11)$ and $26.7 \%(8)$, respectively) $(p>0.05)$. 
Table 2

Characteristics of the postoperative period in the two studied subgroups of group 1

\begin{tabular}{lccc}
\hline \multicolumn{1}{c}{ Indicators } & Subgroup 1.1 $(\boldsymbol{n}=\mathbf{3 0})$ & Subgroup 1.2 $(\boldsymbol{n}=\mathbf{3 0})$ & $\boldsymbol{P}$ \\
\hline $\begin{array}{l}\text { Max increase in body temperature, }{ }^{\circ} \mathrm{C} \\
\text { The day of normalization of body }\end{array}$ & $37.6 \pm 0.04$ & $38.0 \pm 0.08$ & $p<0.001$ \\
temperature & $3.5 \pm 0.4$ & $5.4 \pm 0.6$ & $p<0.05$ \\
Day of postoperative pain relief & $5.2 \pm 0.7$ & $9.4 \pm 1.6$ & $p<0.05$ \\
The average duration of postoperative & $7.2 \pm 0.6$ & $10.2 \pm 1.0$ & $p<0.05$ \\
pain, days & &
\end{tabular}

The analysis of complaints showed that in $85 \%$ (i.e., 51) cases of patients of both subgroups complained of pain in the lower abdomen, in $53.3 \%$ (32) - for bloody discharge from the genital tract of varying intensity, in $46.7 \%$ (28) - for malaise, weakness, dizziness and fainting. Menstrual irregularities were observed in $41.7 \%$ of cases (i.e., in 25 women), dyspepsia - in $31.7 \%$ (19), fever - in $8.3 \%$ (5).

In addition, it should be noted that in subgroup 2.1 in $6.7 \%$ (i.e., 2 women) patients had hemorrhagic shock, and in subgroup 2.2 this figure was $13.3 \%$ (i.e., 4 women) $(p>0.05)$.

As in subgroups of group 1, in group 2 the laparoscopic method was used in subgroup 2.1, and in subgroup 2.2 surgery was performed by laparotomy. In addition, these subgroups were also analyzed for the most informative indicators of tics, such as: average duration of surgery and intraoperative blood loss (Table 3).

Table 3

Characteristics of the postoperative period in the two studied subgroups of group 2

\begin{tabular}{cccc}
\hline Indicators & Subgroup 2.1 $(\boldsymbol{n}=\mathbf{3 0})$ & Subgroup 2.2 $(\boldsymbol{n}=\mathbf{3 0})$ & $\boldsymbol{P}$ \\
\hline Duration of operation, min & $39.8 \pm 2.1$ & $59.1 \pm 2.5$ & $p<0.001$ \\
Intraoperative blood loss, $\mathrm{ml}$ & $189.1 \pm 19.8$ & $388.5 \pm 37.2$ & $p<0.05$
\end{tabular}

An integral criterion in choosing the method of surgical treatment is the duration of the pain response in the postoperative period. Thus, this indicator in subgroup 2.1 was $5.1 \pm 0.3$ days, which was significantly less than in subgroup 2.2, namely $7.1 \pm 0.3$ days $(p<0.05)$.

Analysis of postoperative complications in the compared subgroups of operated patients showed that the frequency of postoperative complications in subgroup 2.1 was $3.3 \%$ (i.e., 1 case), and in $2.2-23.3 \%$ (7 cases), respectively.

All this gives grounds to note that the laparoscopic method of treatment could increase the effectiveness of surgical treatment, reduce the duration of surgery, surgical blood loss and improve the postoperative period.

Also, to achieve this goal, the course of operations and postoperative period in subgroups 3.1 and 3.2 of group 3, which included women with such urgent pathology as torsion of the ovarian cyst leg (43.3\% (13) in subgroup 3.1 and $60 \%$ (18)) were analyzed - in 3.2) and impaired blood supply to uterine tumors, represented mainly by degeneration of the myoma $-56.7 \%$ (17) in subgroup 3.1 and $40 \%(12)-$ in $3.2(p>0.05)$. In addition, in subgroup 3.1 in $20 \%$ of patients (i.e., 6 women), and in subgroup 3.2 in $13.3 \%$ of patients (i.e., 4 women) there was such a complication as pelvioperitonitis.

Given the above in subgroup 3.1 by laparoscopy was performed in $23.3 \%$ (i.e., 7 women) patients subtotal hysterectomy, $33.3 \%$ (i.e., 10 women) patients - myomectomy and $43.3 \%$ (i.e., 13) patients - adnexectomy. In contrast to the previously mentioned subgroup, surgical interventions in subgroup 3.2 were performed by laparotomy: $16.7 \%$ (i.e., 5 women) patients underwent subtotal hysterectomy, $23.3 \%$ (i.e., 7 women) patients underwent myomectomy and $60 \%$ (i.e., in 18) patients - adnexectomy $(p>0.05)$.

The duration of operations in the compared subgroups was $62.1 \pm 4.9$ and $89.6 \pm 7.8$ minutes, respectively $(p<0.01)$, and blood loss was $101.7 \pm 10.1 \mathrm{ml}$ and $283.3 \pm 12.9 \mathrm{ml}(p<0.001)$.

As in the above subgroups 1 and 2 groups, the average postoperative pain relief was $6.9 \pm 0.7$ days for patients in subgroup 3.1 and $9.9 \pm 0.6$ days for $3.2(p<0.01)$. 
Thus, modern endoscopic technologies, in particular the use of laparoscopy in the treatment of urgent genital pathologies, can improve the effectiveness of both surgical treatment and the postoperative period.

\section{Discussion of research results}

In the structure of surgical interventions in gynecology the leading place is occupied by various urgent conditions: ectopic pregnancy, apoplexy and trophic disorders of both ovaries and uterus $[5,6]$. The result of early diagnosis and the choice of the right tactics for their treatment entails a further state of reproductive health of women. And as you know, this is the key to the normal development and functioning of the nation. In gynecology, as in all surgery, there are different methods of surgical treatment. Given the desired result, which includes the least minimally invasive, more and more obstetricians and gynecologists prefer laparoscopy instead of laparotomy. However, there is still a debate about the effectiveness of different methods of surgical treatment.

To this end, three groups were analyzed, which included women with emergencies such as: aPID; intra-abdominal bleeding as a result of ectopic pregnancy and ovarian apoplexy; impaired blood supply to tumors of the internal genitalia (uterus and ovaries).

According to the literature, there is an increase in the frequency of acute aPID. These include endometritis, salpingo-oophoritis, tuboovarian formations and pelvioperitonitis [7, 8]. The results showed that in both study subgroups, $50 \%$ (i.e., 30) of women were diagnosed with unilateral or bilateral pyosalpinx, $20 \%$ (12) of patients with right-sided or left-sided pyovarium, and $30 \%$ (18) of patients were diagnosed purulent tuboovarian formations. All these pathological processes in $53.3 \%$ (32) of women were complicated by pelvioperitonitis.

Analysis of the literature has shown that women's complaints and clinical manifestations of acute aPID are quite diverse - from abdominal pain to dysuric phenomena [9, 10]. The same situation was observed during the study. It was found that in all studied women of the first group the body temperature was above normal (in $100 \%$ (i.e., 60 patients)), and in most of them there were lower abdominal pain (98.3\% (i.e., 59 patients)). In addition, $60 \%$ of patients (i.e., 36 patients) had general weakness, and $40 \%$ of women (i.e., 24 patients) complained of bowel incontinence and $6.7 \%$ of patients (i.e., 4 patients) complained of dysuria.

As mentioned above, laparoscopy refers to minimally invasive treatments in surgical practice, in contrast to surgical interventions by laparotomy [11, 12]. This is confirmed by the data obtained during the study in group 1. The average duration of operations in subgroups 1.1 and 1.2 was $50.4 \pm 7.2$ and $80.3 \pm 10.1$ minutes, respectively, and intraoperative blood loss averaged $210.7 \pm 31.4 \mathrm{ml}$ and $375.9 \pm 44$ in the compared subgroups, $5 \mathrm{ml}$, respectively $(p<0,01)$. These data showed that the worst indicators were in subgroup $1.2(p<0.05)$.

Other indicators that characterize the effectiveness and minimally invasiveness of the treatment method are the course of the postoperative period, in particular the nature and duration of fever, pain $[13,14]$. After analyzing the postoperative period, it was found that the most acceptable method is also laparoscopy. The maximum temperature rose after surgery in patients of subgroup 1.1 was $37.6 \pm 0.04{ }^{\circ} \mathrm{C}$ in contrast to subgroup $1.2-38.0 \pm 0.08{ }^{\circ} \mathrm{C}(p<0.001)$. As for the day of normalization of body temperature, it averaged $3.5 \pm 0.4$ and $5.4 \pm 0.6$ days, respectively $(p<0.05)$. In patients of subgroup 1.1 the day of postoperative pain relief and the average duration of the postoperative period was significantly lower than in subgroup 1.2 (5.2 \pm 0.7 and 9.4 \pm 1.6; $7.2 \pm 0.6$ and $10.2 \pm 1.0$ days, respectively) $(p<0.05)$.

Pathological conditions such as ectopic pregnancy and ovarian apoplexy are also dangerous for a woman's health, as they are one of the causes of intrahepatic bleeding and hemorrhagic shock as a consequence $[15,16]$. To treat these emergencies, as in subgroups of group 1, group 2, the laparoscopic method was used in subgroup 2.1, and in subgroup 2.2, surgery was performed by laparotomy. The results of operations showed that its average duration was $39.8 \pm 2.1$ minutes in subgroup 2.1 and $59.1 \pm 2.5$ minutes in subgroup $2.2(p<0.001)$, and intraoperative blood loss averaged $189.1 \pm 19,8$ and $388.5 \pm 37.2 \mathrm{ml}$, respectively $(p<0.05)$.

Since the duration of the pain response in the postoperative period is an integral criterion in the choice of surgical treatment $[17,18]$, this indicator was also analyzed in subgroups of group 2. 
Thus, in subgroup 2.2 it was 1.4 times longer than in subgroup $2.1(p<0.05)$, which indicates that laparoscopic treatment is more acceptable in the treatment of pathologies such as ectopic pregnancy and ovarian apoplexy.

No less important in the early diagnosis and treatment are tumors of the internal genitalia with impaired blood supply, including torsion of the ovarian cyst and degeneration of the myoma, as these conditions are often accompanied by pelvioperitonitis [19,20]. In both subgroups of group 3, the number and type of operations did not differ significantly $(p>0.05)$. The choice of laparoscopic method of treatment is indicated by the average duration of the operation, which in subgroup 3.2, where the laparotomy method was used, was 1.4 times longer compared to subgroup 3.1, where the laparoscopic method was used. The situation is similar with blood loss, where it was 2.8 times greater in the subgroup in which patients underwent surgical treatment by laparotomy. Another important criterion was the time of postoperative pain relief, which was 1.4 times longer in subgroup 3.2 compared to subgroup 3.1.

Study limitations. The study did not involve women with third-degree obesity, with a pronounced connective tissue process and with severe somatic diseases.

Prospects for further research. Improving the effectiveness of surgical treatment of the pelvic organs in the postmenopausal period.

\section{Conclusions}

The results of the study showed that:

- the average duration of laparotomy operations in patients with aPID was 1.6 times longer than the laparoscopic method;

- intraoperative blood loss in patients with aPID who underwent laparotomy was 1.8 times greater than in patients who underwent laparoscopy;

- in the postoperative period, the maximum temperature rise in patients with aPID was significantly higher in the subgroup where the laparotomy method was used, compared with another group where the laparoscopic method of surgical treatment was used $\left(38.0 \pm 0.08^{\circ} \mathrm{C}\right.$ vs. $\left.37.6 \pm 0.04^{\circ} \mathrm{C}\right)(p<0.001)$;

- the day of normalization of body temperature was 1.5 times greater in subgroup 1.2 compared to subgroup 1.1;

- the average duration of the postoperative period in patients of subgroup 1.1 was significantly shorter compared to subgroup $1.2(7.2 \pm 0.6$ and $10.2 \pm 1.0$ days $)(p<0.05)$;

- the average duration of operations in subgroup 2.2 , which included women with IAB, and which used the laparotomy method, was 1.4 times longer than in subgroup 2.1, where interventions were performed by laparoscopy $(p<0.001)$;

- intraoperative blood loss on average was also significantly lower in subgroup 2.1, which included women with IAB, and which used laparotomy, compared with subgroup 2.2, which used laparotomy surgical treatment (189.1 \pm 19.8 and 388, $5 \pm 37.2 \mathrm{ml}$, respectively) $(p<0.05)$;

- the duration of pain in the postoperative period in subgroup 2.2 was significantly longer than in subgroup 2.1 ( $7.1 \pm 0.3$ vs. $5.1 \pm 0.3$ days $)(p<0.05)$;

- the average duration of operations was significantly longer in subgroup 3.2, which included women with tumors of the internal genitalia with impaired blood supply and who underwent laparotomy surgery than in the subgroup in which women underwent laparoscopy, namely in 1.4 times;

- blood loss during surgery in subgroup 3.2 was significantly greater than in group 3.1 (283.3 \pm 12.9 vs. $101.7 \pm 10.1 \mathrm{ml}$, respectively) $(p<0.001)$;

- in subgroup 3.2, where the laparotomy method of surgical treatment was used, the day of postoperative pain relief was also significantly longer compared to subgroup 3.1, where laparoscopy was used $(9.9 \pm 0.6$ vs. $6.9 \pm 0.7$ days $)(p<0.01)$.

\section{Conflict of interest}

The authors declare that they have no conflicts of interest.

\section{Financing}

The authors declare there was no financial support. 


\section{References}

[1] Lawani, O. L., Anozie, O. B., Ezeonu, P. O. (2013). Ectopic pregnancy: a life-threatening gynecological emergency. International Journal of Women's Health, 5, 515-521. doi: http://doi.org/10.2147/ijwh.s49672

[2] Kalogera, E., Bakkum-Gamez, J. N., Jankowski, C. J., Trabuco, E., Lovely, J. K., Dhanorker, S. et. al. (2013). Enhanced Recovery in Gynecologic Surgery. Obstetrics \& Gynecology, 122 (2), 319-328. doi: http://doi.org/10.1097/aog.0b013e31829aa780

[3] Thepsuwan, J., Huang, K.-G., Wilamarta, M., Adlan, A.-S., Manvelyan, V., Lee, C.-L. (2013). Principles of safe abdominal entry in laparoscopic gynecologic surgery. Gynecology and Minimally Invasive Therapy, 2 (4), 105-109. doi: http:// doi.org/10.1016/j.gmit.2013.07.003

[4] Alobaid, A., Memon, A., Alobaid, S., Aldakhil, L. (2013). Laparoscopic Management of Huge Ovarian Cysts. Obstetrics and Gynecology International, 2013, 1-4. doi: http://doi.org/10.1155/2013/380854

[5] Rana, P., Kazmi, I., Singh, R., Afzal, M., Al-Abbasi, F. A., Aseeri, A. et. al. (2013). Ectopic pregnancy: a review. Archives of Gynecology and Obstetrics, 288 (4), 747-757. doi: http://doi.org/10.1007/s00404-013-2929-2

[6] Elson, C. J., Salim, R., Potdar, N., Chetty, M., Ross, J. A., Kirk, E. J. (2016). Diagnosis and management of ectopic pregnancy. BJOG: An International Journal of Obstetrics and Gynaecology, 123 (13), e15-e55. doi: http://oi.org/10.1111/1471-0528.14189

[7] Carrascosa, P., Capuñay, C., Sueldo, C. E., Baronio, J. M. (2014). Tubal pathology. CT Virtual Hysterosalpingography. Cham: Springer, 191-202. doi: http://doi.org/10.1007/978-3-319-07560-0_9

[8] Göksever Çelik, H., Çelik, E., Yüksel, S., Baştu, E., Ark, H. C. (2017). Different Surgical Techniques in Tubo-ovarian Abscess Management. Gynecology Obstetrics \& Reproductive Medicine, 23 (1), 32-36. doi: http://doi.org/10.21613/gorm.2016.639

[9] Kim, H. Y., Yang, J. I., Moon, C. (2014). Comparison of severe pelvic inflammatory disease, pyosalpinx and tubo-ovarian abscess. Journal of Obstetrics and Gynaecology Research, 41 (5), 742-746. doi: http://doi.org/10.1111/jog.12617

[10] Ross, J., Chacko, M. (2019). Pelvic inflammatory disease: Clinical manifestations and diagnosis. Available at: https://www. uptodate.com/contents/pelvic-inflammatory-disease-clinical-manifestations-and-diagnosis

[11] Jiang, X., Shi, M., Sui, M., Wang, T., Yang, H., Zhou, H., Zhao, K. (2019). Clinical value of early laparoscopic therapy in the management of tubo-ovarian or pelvic abscess. Experimental and therapeutic medicine, 18 (2), 1115-1122. doi: http://doi.org/ 10.3892/etm.2019.7699

[12] Snyman, L., Makulana, T., Makin, J. D. (2017). A randomised trial comparing laparoscopy with laparotomy in the management of women with ruptured ectopic pregnancy. South African Medical Journal, 107 (3), 258-263. doi: http://doi.org/10.7196/ samj.2017.v107i3.11447

[13] Eltabbakh, G. H., Charboneau, A. M., Eltabbakh, N. G. (2008). Laparoscopic surgery for large benign ovarian cysts. Gynecologic Oncology, 108 (1), 72-76. doi: http://doi.org/10.1016/j.ygyno.2007.08.085

[14] Joo, J., Moon, H. K., Moon, Y. E. (2019). Identification of predictors for acute postoperative pain after gynecological laparoscopy (STROBE-compliant article). Medicine, 98 (42), e17621. doi: http://doi.org/10.1097/md.0000000000017621

[15] Alkatout, I., Honemeyer, U., Strauss, A., Tinelli, A., Malvasi, A., Jonat, W. et. al. (2013). Clinical Diagnosis and Treatment of Ectopic Pregnancy. Obstetrical \& Gynecological Survey, 68 (8), 571-581. doi: http://doi.org/10.1097/ogx.0b013e31829cdbeb

[16] Kirk, E., Bottomley, C., Bourne, T. (2013). Diagnosing ectopic pregnancy and current concepts in the management of pregnancy of unknown location. Human Reproduction Update, 20 (2), 250-261. doi: http://doi.org/10.1093/humupd/dmt047

[17] Schwenkglenks, M., Gerbershagen, H. J., Taylor, R. S., Pogatzki-Zahn, E., Komann, M., Rothaug, J. et. al. (2014). Correlates of satisfaction with pain treatment in the acute postoperative period: Results from the international PAIN OUT registry. Pain, 155 (7), 1401-1411. doi: http://doi.org/10.1016/j.pain.2014.04.021

[18] Jarrell, J., Ross, S., Robert, M., Wood, S., Tang, S., Stephanson, K., Giamberardino, M. A. (2014). Prediction of postoperative pain after gynecologic laparoscopy for nonacute pelvic pain. American Journal of Obstetrics and Gynecology, 211 (4), 360. e1-360.e8. doi: http://doi.org/10.1016/j.ajog.2014.04.010

[19] Ding, D.-C., Huang, C., Hong, M.-K. (2017). A review of ovary torsion. Tzu Chi Medical Journal, 29 (3), 143-147. doi: http:// doi.org/10.4103/tcmj.tcmj_55_17

[20] Asfour, V., Varma, R., Menon, P. (2015). Clinical risk factors for ovarian torsion. Journal of obstetrics and gynaecology, 35 (7), 721-725.

Received date 25.12.2021

(C) The Author(s) 2022

Accepted date 25.01.2022

This is an open access article

Published date 31.01.2022 under the Creative Commons CC BY license

How to cite: Pikul, Iu., Lubkovska, O. (2022). Emergency treatment in gynecology using different methods of surgery. EUREKA: Health Sciences, 1, 3-9. doi: http://doi.org/10.21303/2504-5679.2022.002276 